\title{
Sonic Hedgehog Is Essential for First Pharyngeal Arch Development
}

\author{
CHIHIRO YAMAGISHI, HIROYUKI YAMAGISHI, JUN MAEDA, TAKATOSHI TSUCHIHASHI, KATHRYN IVEY, \\ TONGHUAN HU, AND DEEPAK SRIVASTAVA \\ Department of Pediatrics [C.Y., H.Y., J.M., T.T.], Keio University School of Medicine, Tokyo 160-8582, Japan; Departments of
Pediatrics and Molecular Biology [C.Y., H.Y., J.M., T.T., K.I., T.H., D.S.], University of Texas Southwestern Medical Center, \\ Dallas, Texas 75390
}

\begin{abstract}
The secreted protein sonic hedgehog (Shh) is essential for normal development of many organs. Targeted disruption of Shh in mouse leads to near complete absence of craniofacial skeletal elements at birth, and mutation of $\mathrm{SHH}$ in human causes holoprosencephaly (HPE), frequently associated with defects of derivatives of pharyngeal arches. To investigate the role of Shh signaling in early pharyngeal arch development, we analyzed Shh mutant embryos using molecular markers and found that the first pharyngeal arch (PA1) was specifically hypoplastic and fused in the midline, and remaining arches were well formed at embryonic day (E) 9.5. Molecular analyses using specific markers suggested that the growth of the maxillary arch and proximal mandibular arch was severely defective in Shh-null PA1, whereas the distal mandibular arch was less affected. TUNEL assay revealed an increase in the number of apoptotic signals in PA1 of Shh mutant embryos. Ectodermal expression of fibroblast growth factor $(F g f)-8$, a cell survival factor for pharyngeal arch mesenchyme, was downregulated in the PA1 of Shh mutants. Consistent with this observation, downstream transcriptional targets of Fgf8 signaling in neural crest-derived mesenchyme, including Barx1, goosecoid, and $D l x 2$, were also down-regulated in Shh-null PA1. These results demonstrate that epithelial-mesenchymal signaling and transcriptional events coordinated by Shh, partly via Fgf8, is essential for cell survival and tissue outgrowth of the developing PA1. (Pediatr Res 59: 349-354, 2006)
\end{abstract}

$\mathrm{P}$ haryngeal arches are bilaterally symmetric ventral structures that develop in a segmental fashion along the anteroposterior axis during embryogenesis (1). The first pharyngeal arch (PA1), which in mammals develops into jaws, lateral skull wall, teeth, middle ear, and part of the tongue and other soft tissue derivatives, is formed as the most rostral and earliest pharyngeal arch beginning at E8.25 in the mouse embryo. This arch rapidly increases in size as it is populated by mesenchyme derived from cranial NCC and develops into the mandibular and maxillary arches at E9.5.

Received June 21, 2005; accepted October 25, 2005

Correspondence: Deepak Srivastava, M.D., Gladstone Institute of Cardiovascular Disease and Department of Pediatrics, University of California San Francisco, 1650 Owens Street, San Francisco, CA 94158; e-mail: dsrivastava@gladstone.ucsf.edu

D.S. was supported by grants from the NHLBI/NIH, March of Dimes Birth Defects Foundation, and American Heart Association. H.Y. was supported by grants from Pfizer funds for development and growth, and the Japanese Ministry of Education and Science.

DOI: 10.1203/01.pdr.0000199911.17287.3e
Appropriate epithelial-mesenchymal signaling is essential for proper development of the pharyngeal arches. Genetic analyses in mice provide evidence that numerous homeobox genes, including Msx, Dlx, goosecoid (Gsc), and Prx, and other transcription factors, such as Hand2, are expressed in pharyngeal arch mesenchyme and play essential roles in development of PA1 $(1,2)$.

Members of the Fgf family, particularly Fgf8, are epithelial signals that regulate gene expression during PA1 development $(3,4)$. Inactivation of mouse Fgf8 specifically in PA1 epithelium revealed that Fgf8 promotes mesenchymal cell survival and induces a developmental program required for PA1 morphogenesis (5). Members of the Bmp family also have important roles in outgrowth of PA1 (4). PA1 development appears very sensitive to the level of Bmp signaling during the initial outgrowth phase, and the level of Bmp signaling is tightly regulated by various factors (6).

The secreted protein Shh, a vertebrate ortholog of the Drosophila segment polarity gene, Hedgehog, is essential for normal development of many organs and is implicated as a cause of HPE. Shh is expressed in the pharyngeal arch epithelium and targeted disruption of $S h h$ in mouse leads to near complete absence of craniofacial skeletal elements along with multiple organ defects (7). Recent studies using chick and mouse embryos have suggested that Shh may play a role in NCC development and pharyngeal pouch patterning (8-10).

Here, we analyzed the developing pharyngeal arches in Shh mutant embryos and found that Shh may be required for outgrowth of PA1 by regulating epithelial-mesenchymal interactions partly via Fgf signaling pathways that ultimately promote cell survival of mesenchymal cells in PA1.

\section{METHODS}

Mouse genetic studies. Mice heterozygous for the Shh mutation or the Pax3-Cre transgene were previously described (7,11). Pax3-Cre transgenic mice were crossed with lacZ reporter mice (Rosa26 reporter: R26R) (12).

Abbreviations: Bmp, bone morphogenic protein; E, embryonic day;Fgf, fibroblast growth factor; HPE, holoprosencephaly; Gsc, goosecoid; NCC, neural crest cells; PA1, first pharyngeal arch; R26R, Rosa26 reporter; Shh, sonic hedgehog; SMMCI, solitary median maxillary central incisor 
These mice were crossed and pregnant mothers were killed at E8.5-9.5 to obtain $S h h^{-1} /$ Pax3-Cre:R26R and $S h h^{-1}$ embryos. This study has been approved by University of Texas Animal Care and Use Committee.

$\boldsymbol{\beta}$-Galactosidase staining. Embryos were dissected, fixed in 2\% paraformaldehyde/PBS with phenol red, and stained in Xgal solution as described previously (13).

Whole-mount and section RNA in situ hybridization. Whole-mount RNA in situ hybridizations were performed using digoxigenin-labeled antisense riboprobes as described previously (13). Section RNA in situ hybridizations were performed on paraffin-embedded sections of mouse embryos as described previously (14).

TUNEL analysis and cell proliferation assay. TUNEL analysis was performed on paraffin sections using apoptosis detection kit (ApopTag, Intergen, Purchase, NY) following manufacturer's protocol with the blue fluorescent DAPI nucleic acid stain (Molecular Probes, Eugene, OR). Cell proliferation assay was performed on paraffin section by immunohistochemistry with purified mouse anti-human Ki-67 antibody $(2.5 \mu \mathrm{g} / \mathrm{mL})(\mathrm{BD}$ Pharmingen, San Diego, CA) using VECTASTAIN ABC kit (Vector Laboratories, Burlingame, CA) following manufacturer's protocol.

Bead experiments on cultured first pharyngeal arch explants. The first pharyngeal arches were dissected in DMEM (Invitrogen, Carlsbad, CA) from E9.5 embryos and cultured on membrane filter (Transwell, Corning, Palo Alto, CA). Bead experiments were performed as described previously (15). Explants were washed in PBS and fixed in $4 \%$ paraformaldehyde overnight at $4{ }^{\circ} \mathrm{C}$, and then subjected to whole-mount in situ hybridization for $F g f 8$ expression.

\section{RESULTS}

Hypoplasia and midline fusion of the first pharyngeal arches in Shh mutant mice. To examine how Shh signaling might regulate early pharyngeal arch development, we analyzed the developing pharyngeal arches in Shh mutant embryos. At E9.5, Shh mutant embryos were grossly thinner and smaller in the head with hypoplasia of PA1 compared with wild-type littermates, but were similar in length (cranial to caudal) (Fig. 1). Morphometrics of PA1 in wild-type versus Shh mutant embryos demonstrated sizes of $0.405 \pm 0.033 \mathrm{~mm}$ versus $0.281 \pm 0.039$ $\mathrm{mm}$ along the dorsal-ventral axis, respectively, and $0.313 \pm$ $0.019 \mathrm{~mm}$ versus $0.142 \pm 0.015 \mathrm{~mm}$ along the anteroposterior axis, respectively ( $p<0.05$ along both axes). In addition to morphologic and histologic indications, hypoplasia of PA1 was demonstrated using a unique property of the Pax3-Cre/R26R transgenic mouse line. In this mouse line, a population of NCC and their progeny can be marked with lac $Z$ by Cre-mediated recombination under control of the $\operatorname{Pax} 3$ promoter, which drives transcription specifically in the early migratory NCC that populate the second to fourth pharyngeal arches, but not PA1 (Fig. 1, $B$ and $C$ ). We crossed these transgenic mice into the $S h h$-null background, and stained the obtained embryos with X-gal. The hypoplastic arch in $S h h$ mutants was PA1 as marked by exclusion of lacZ expression (Fig. $1 G$ ). In frontal view, PA1 of Shh mutants appeared as a single hypoplastic arch structure with the bilateral PA1s being fused in the midline anterior to the second pharyngeal arches (Fig. $1 H$ ). This analysis also demonstrated relatively normal size and patterning of second to fourth pharyngeal arches at E9.5, indicating that NCC migration into second to fourth arches was grossly normal in Shh mutants (Fig. 1, $G$ and $H$ ). Histologic analyses suggested a small and fused single arch structure in the midline, absence of the maxillary component and hypoplasia of the mandibular component of PA1 in Shh mutants (Fig. 1I) compared with wild-type embryos (Fig. 1D), although bilaterally symmetric PA1 were initially developed at E8.5 in Shh mutant embryos (Fig. 1J).

To elucidate a mechanism for the PA1 defect in Shh mutant embryos, we performed in situ hybridization using markers specific for PA1 development. Bmp4 is normally expressed in numerous embryonic domains, including the distal epithelium of the mandibular, maxillary, and frontonasal region, as well as the cardiac outflow tract at E9.5 (Fig. 2, $A$ and $M)(16,17$ ). In $S h h$ mutant embryos, the expression of Bmp4 was specifically absent in the maxillary epithelium, whereas its expression in the mandibular and frontonasal epithelium, and the cardiac outflow tract was intact (Fig. 2, D and P). Mhox and

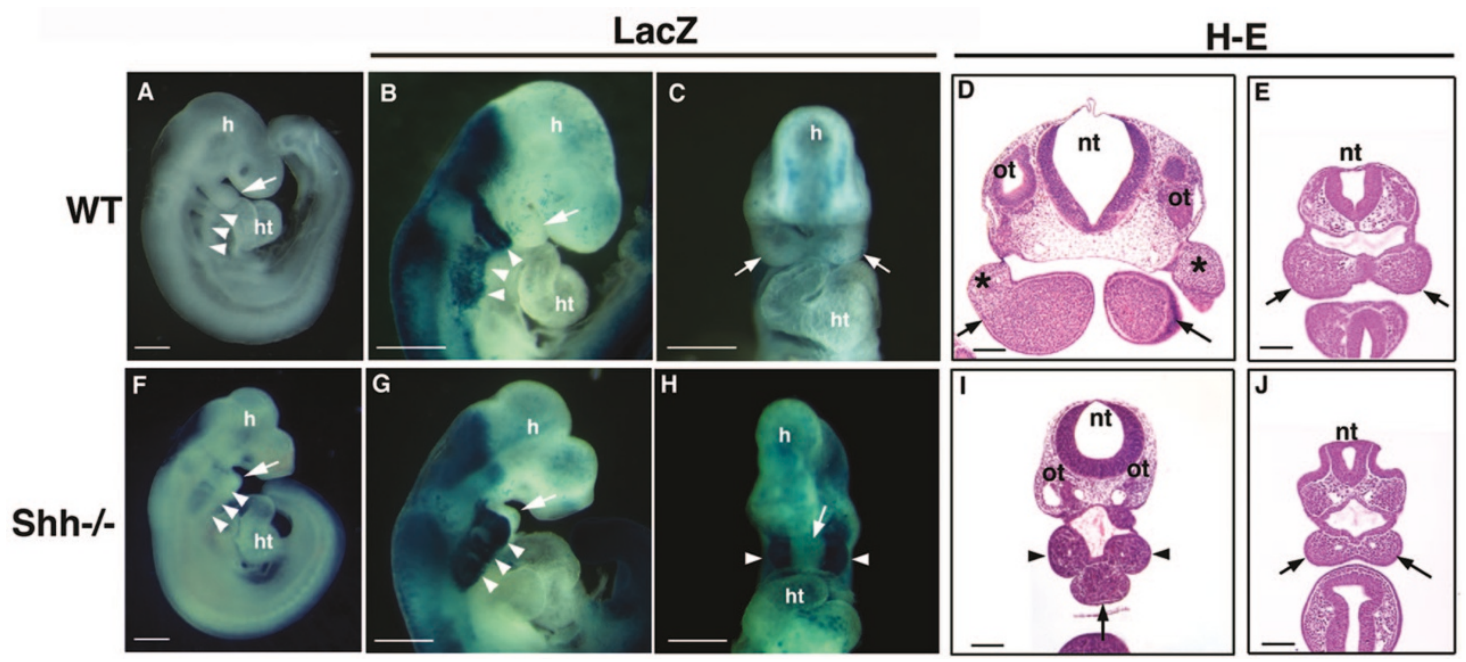

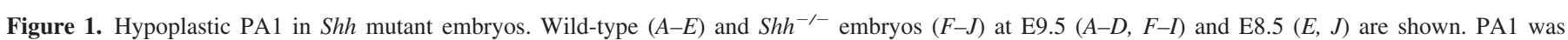

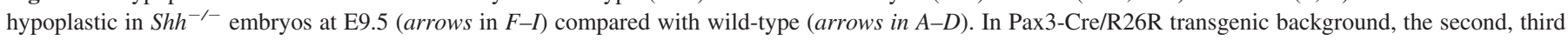

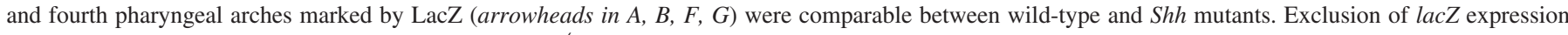

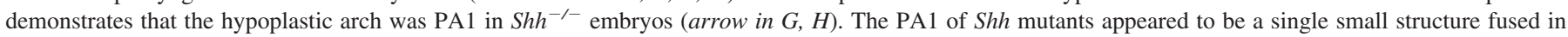

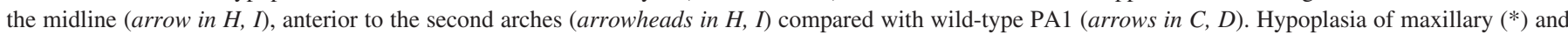

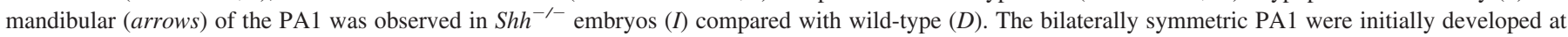

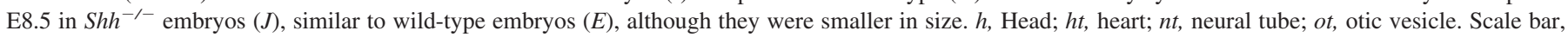
$0.5 \mathrm{~mm}(A-C, F-H) ; 0.1 \mathrm{~mm}(D, E, I, J)$. 

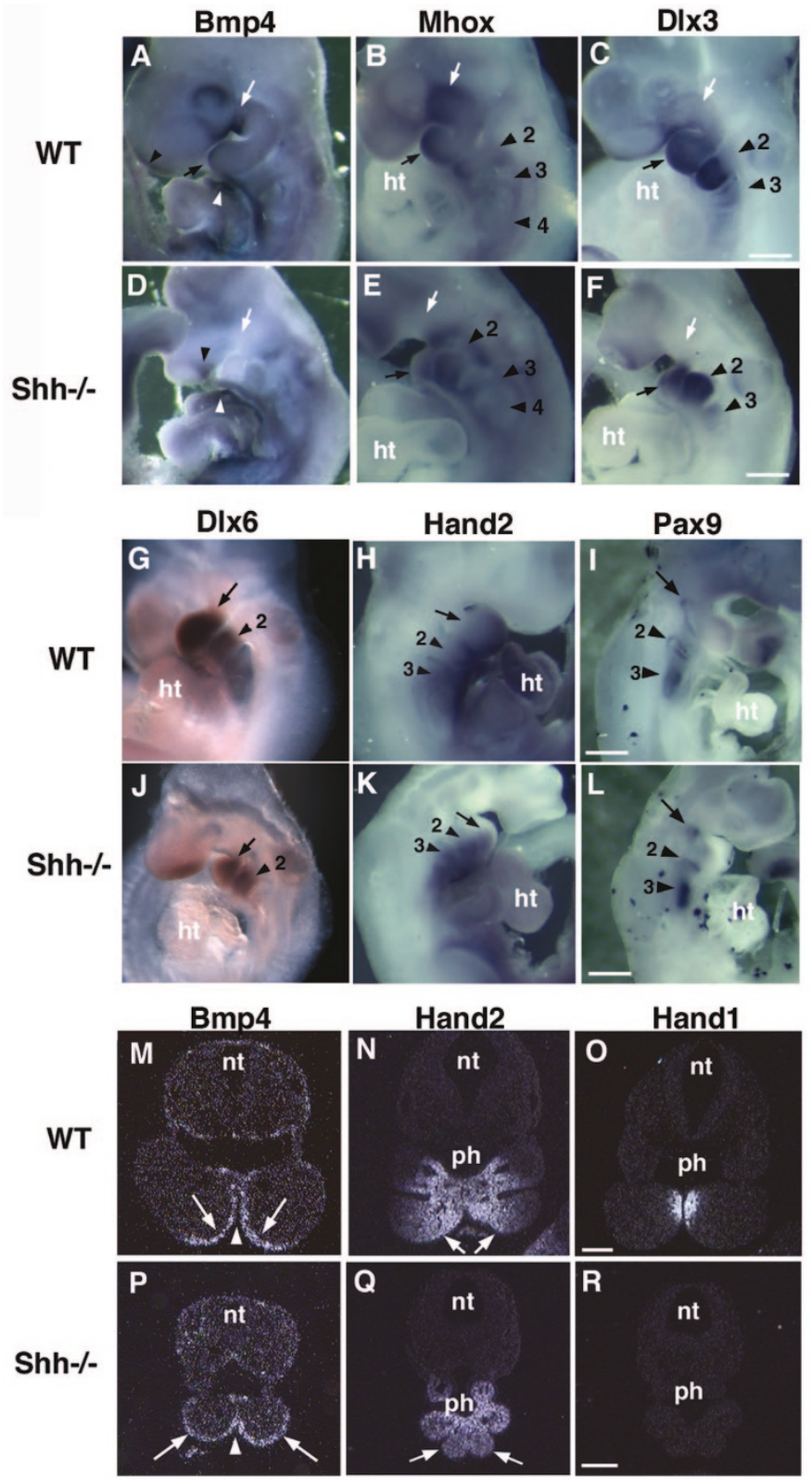

Figure 2. Severe hypoplasia of the maxillary and the proximal mandibular arches, and defective midline development of PA1 in Shh mutants. Wholemount in situ hybridization $(A-L)$ and radioactive section in situ hybridization $(M-R)$ of molecular markers at E9.5 are shown. Bmp4 was expressed in the distal epithelium of the mandibular (black arrow), maxillary (white arrow), frontonasal (black arrowhead) region and cardiac outflow tract (white arrowhead) in wild-type embryos $(A)$, whereas the expression of maxillary epithelium was not detectable in $S_{h} h^{-1}$ embryos $(D)$. Mhox and Dlx3 were expressed in maxillary (white arrow) and mandibular (black arrow) mesenchyme derived from NCC of wild-type embryos $(B, C)$. Their expressions were absent in the maxillary component, but intact in the mandibular component of $S h h^{-1}$ embryos $(E, F)$. Arrowheads with numbers denote the second to fourth pharyngeal arch mesenchyme derived from NCC. Dlx6 and Hand2 were similarly expressed in the mandibular mesenchyme (arrow) in $S h h^{-1}$ embryos ( $\left.J, K\right)$ compared with wild-type embryos $(G, H)$. Pax9 was normally detected in the pharyngeal endoderm (pharyngeal pouches) of Shh mutants at E9.5 (L) compared with wild-type embryos (I). In transverse section, Bmp4 was expressed in the distal epithelium of the mandibular component of wild-type embryos (arrows in M). In Shh ${ }^{-1}$ embryos, Bmp4 was expressed throughout the epithelium of the mandibular arch (arrows in $P$ ). Ectodermal epithelium of right and left mandibular arches appeared to be fused in the midline of $S h h^{--}$embryos (arrowhead in P), in contrast to wild-type embryos where they are separate (arrowhead in $M$ ). Hand 2 is
Dlx3 are expressed in maxillary and mandibular mesenchyme derived from NCC in wild-type embryos at E9.5 (Fig. 2, B and $C)$. Their expressions were undetectable in the maxillary component, but detectable in the mandibular component of Shh mutant embryos (Fig. 2, $E$ and $F$ ). Consistent with these results, the expression of Dlx6 and Hand2, detectable only in the distal mandibular mesenchyme of wild-type embryos at E9.5 (Fig. 2, $G$ and $H$ ), were unaltered in Shh mutant embryos (Fig. 2, $J$ and $K$ ). In transverse sections of the mandibular arch at E9.5, expression of Bmp4 was detectable only in the distal epithelium of wild-type embryos (Fig. $2 M$ ), however, it was detected throughout the PA1 epithelium of Shh mutants (Fig. $2 P$ ), suggesting that the proximal mandibular component might be hypoplastic in the Shh mutant PA1. The Bmp4 expression domain in the medial epithelium of PA1 was absent in mutants and the ectodermal epithelium of right and left PA1 appeared to be continuous in Shh mutant embryos (Fig. 2P), probably reflecting a midline defect of the Shh mutant PA1. A defective proximal mandibular component and midline structure of PA1 in Shh mutants was further demonstrated by the expression pattern of Hand genes in transverse sections at E9.5. Hand2 is normally expressed in mesenchyme of the distal, but not proximal, region of the mandibular arch (Fig. 2, $H$ and $N$ ). In Shh mutant embryos, the expression of Hand2 was homogenously detectable in PA1 (Fig. 2Q), further suggesting that the proximal region of mandibular arch was severely hypoplastic in these mutants. Hand 1 is normally expressed in the medial mesenchyme of PA1 (Fig. 2O). However, no expression of Handl was detectable in Shh mutants (Fig. $2 R$ ), consistent with a loss of midline structure in PA1.

In spite of the PA1 defect, expression of the endodermal marker, $\operatorname{Pax} 9$, was normally detected in the pharyngeal endoderm (pharyngeal pouches) of Shh mutants at E9.5 (Fig. $2 L$ ) compared with wild type embryos (Fig. 2I), suggesting that initial endodermal development and pharyngeal patterning were unaffected in mouse embryos lacking Shh by E9.5. Significant expressions of the NCC-derived mesenchymal cell markers, Mhox (Fig. 2E), Dlx3 (Fig. 2F), Dlx6 (Fig. 2J) and Hand2 (Fig. $2 K$ ) were observed in PA1 as well as other arches of Shh mutant embryos at E9.5, indicating that NCC could, at least in part, migrate and differentiate in PA1. Taken together, Shh signaling may be critical for proper outgrowth of PA1, especially the maxillary arch and the proximal region of mandibular arch, in addition to establishment of the midline structure during PA1 development.

Shh is required for survival of mesenchymal cells in the first pharyngeal arch. To test whether the failure of PA1 outgrowth in Shh mutant embryos might be the result of a cell survival defect, we performed TUNEL assays on tissue sections to mark apoptotic cells. Little apoptosis was detected in PA1 of wild type at E9.0-9.5 (Fig. 3, A-D). In contrast, a

normally expressed in the mesenchyme of the distal (arrows in $H, N$ ), but not proximal, region of the mandibular arches $(N)$. In $S h h^{-1}$ embryos, the expression of Hand2 was homogenously detectable in the fused mandibular arch (arrows in $Q$ ). Hand1 is normally expressed in the medial mesenchyme of mandibular arches $(O)$, but it was not detectable in $S h h^{-/}$embryos $(R) . h t$, Heart; $n t$, neural tube; $p h$, pharynx. Scale bar, $0.3 \mathrm{~mm}(A-L) ; 0.1 \mathrm{~mm}(M-R)$. 

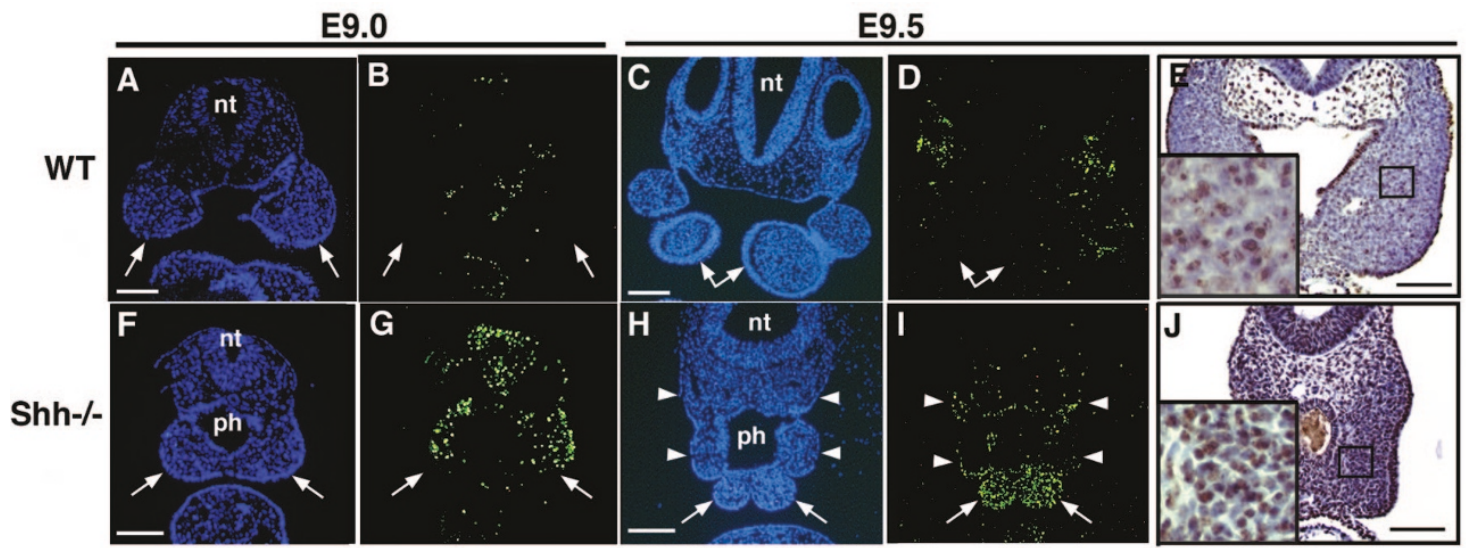

Figure 3. Enhanced apoptosis in the PA1 of $S h h$ mutant embryos. Transverse sections of wild-type $(A-E)$ and $S h h^{-/}$embryos $(F-J)$ at E9.0 $(A, B, F, G)$ and E9.5 $(C-E, H-J)$ were analyzed by TUNEL assay $(B, D, G, I)$ and counter-stained with DAPI $(A, C, F, H)$, and assayed for cell proliferation by immunohistochemistry using anti-Ki-67 antibody $(E, J)$. Enhanced apoptotic signals were observed in mesenchyme of $S h h^{-1-}$ PA1 (arrows), but not in the second or third pharyngeal arches (arrowheads), beginning from the maxillary and the proximal mandibular region at E9.0 $(G)$ and extending throughout the PA1 at E9.5 $(I)$, compared with wild type ( $B$ and $D$, respectively). Cell proliferation appeared relatively normal in PA1 of Shh mutants $(J)$ compared with wild-type embryos $(E)$. Inset is a higher magnification of the boxed area. $n t$, Neural tube; $p h$, pharynx. Scale bar: $0.1 \mathrm{~mm}$

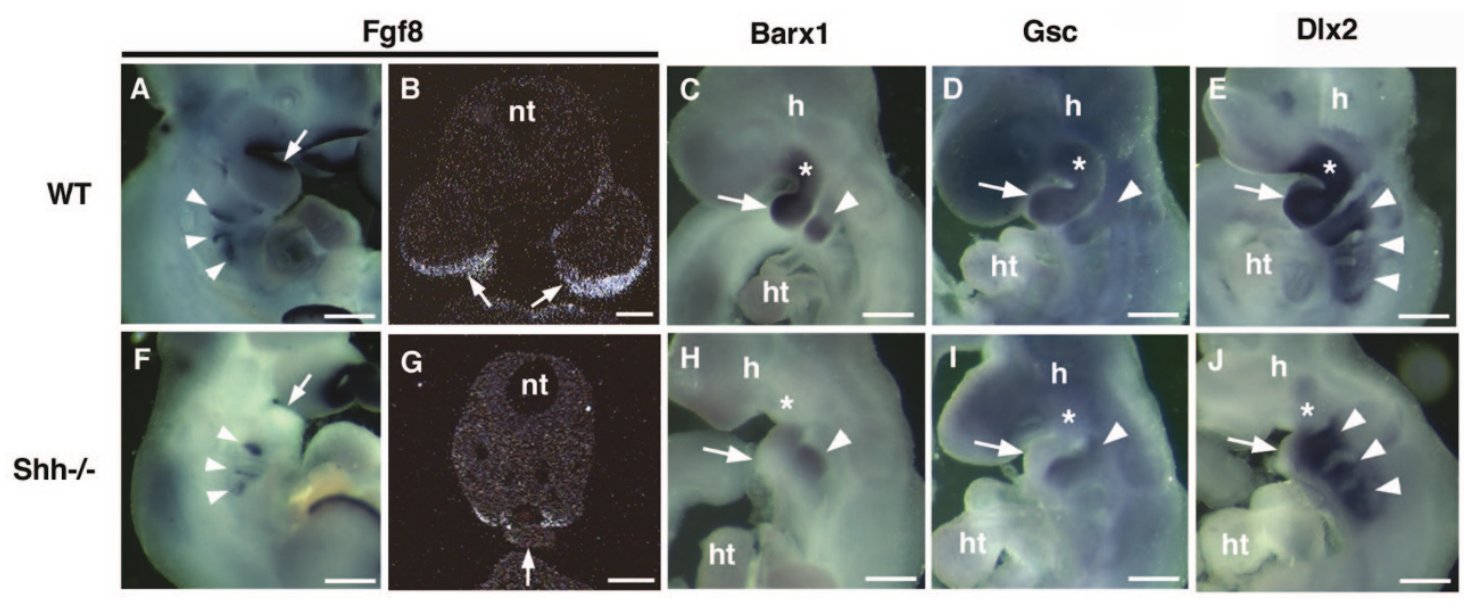

Figure 4. Requirement of Shh for Fgf signaling during epithelial-mesenchymal interactions in the PA1. Whole-mount and section in situ hybridization of wild-type $(A-E)$ and $S h h^{-/}$embryos $(F-J)$ at E9.5 are shown. $F g f 8$ was expressed in the PA1 ectoderm of wild-type embryos (arrows in A, B) but was down-regulated in Shh mutants (arrows in F, G), with normal expression in the second to fourth pharyngeal arches (arrowheads in A, F). Barxl, Gsc, and Dlx2 were normally expressed in mesenchymal cells in the PA1 (arrows in $C-E$ ) and other pharyngeal arches at E9.5 (arrowheads in $C-E$ ). In $S h h^{-1-}$ embryos, they were specifically down-regulated in PA1 (arrows in $H-J$ ), with normal expression in other arches (arrowheads in H-J). Down-regulation of Barxl, Gsc, and $D l x 2$ were noted in the maxillary component $(*)$ of mutant PA1 $(H-J)$ compared with wild type $(C-E)$. $h$, Head; $h t$, heart; $n t$, neural tube. Scale bar: 0.3 mm $(A, C-F, H-J), 0.1 \mathrm{~mm}(B, G)$.

progressive increase in apoptosis was detected in Shh mutants, mainly in the proximal region at E9.0 (Fig. 3, $F$ and $G$ ), and throughout PA1 mesenchyme at E9.5 (Fig. 3, $H$ and $I$ ). Enhanced apoptotic signals were detectable only in PA1, but not in the second and third arches at E9.5 (Fig. 3, $H$ and $I$ ). In contrast, cell proliferation assayed by immunohistochemistry using anti-Ki-67 antibody appeared relatively normal in PA1 of Shh mutants at E9.5 (Fig. 3J) compared with wild-type embryos (Fig. 3E). These results suggest that hypoplasia of PA1 in Shh mutants at E9.5 is mainly due to apoptosis of a substantial proportion of the cells that normally give rise to PA1.

Shh is required for epithelial-mesenchymal signaling in the first pharyngeal arch. In addition to Bmp4, Fgf8 is a critical signaling molecule that transmits survival signals from the epithelial cells to the adjacent mesenchyme (4-6). In contrast to Bmp4, which is expressed in the epithelia, albeit in an abnormal pattern (Fig. 2, $D$ and $P$ ), $F g f 8$ was specifically down-regulated in the ectodermal epithelium of PA1 in Shh mutants at E9.5 (Fig. 4, $F$ and $G$ ), coinciding with the extensive apoptosis observed. The expression of Fgf8 in the second and third pharyngeal regions was normal at this stage.

Because Fgf8 was down-regulated in Shh mutants, we examined expression of several transcription factors that are downstream of Fgf8 signaling in the pharyngeal arch mesenchyme $(2,5)$. Genes encoding the homeobox transcription factors Barxl, Gsc, and Dlx2 are expressed in NCC-derived mesenchymal cells in the PA1 and other pharyngeal arches (Fig. 4, $C-E$ ), and their expression is dependent upon Fgf8 signaling. Consistent with down-regulation of $F g f 8$, expression of Barxl, Gsc, and Dlx2 were decreased in the PA1 of Shh mutants at E9.5, whereas expressions in other arches were normal (Fig. 4, H-J). These results indicate that the epithelial- 
mesenchymal interactions mediated by Fgf8 signaling were affected in PA1 of Shh mutant embryos.

To determine whether Shh can activate $F g f 8$ expression, we attempted to induce Fgf8 mRNA with Shh-soaked beads in cultured PA1. Compared with the control PA1 cultured with BSA-soaked beads, the expression level of $F g f 8$ was elevated in PA1 cultured with Shh-soaked beads (Fig. 5A). Despite the limitations of this experiment using cultured tissues, it supports the observation in Shh mutants that Fgf8 may function downstream of Shh signaling during PA1 development.

\section{DISCUSSION}

Shh signaling plays a primary role for early PA1 development. In this report, we analyzed pharyngeal arch development in Shh mutant embryos, focusing on PA1, and demonstrated that bilateral PA1 initially form, but they become hypoplastic, resulting in a single fused structure in the midline by E9.5. Exposure to the Shh inhibitor, jervine, at E7.5 frequently leads to only forebrain defects, but no mandibular defects, whereas susceptibility for mandibular defects is highest when pregnant mice are treated around E9.5 (18). A recent study demonstrated that mice lacking Hh-responsiveness specifically in cranial NCC showed a hypoplastic PA1 without mid- or forebrain defects at E11.5 (9). Taken together, the PA1 defect in Shh mutants at E9.5 presented in this study is likely to be a primary defect resulting from lack of Shh signaling.

Our analyses did not address whether NCC migration into PA1 was altered in Shh mutant embryos. Significant expression of the NCC-derived mesenchymal cell markers, Mhox, Dlx3, Dlx6, and Hand2, was observed in PA1 as well as other arches of Shh mutant embryos at E9.5, indicating that NCC could, at least in part, migrate and differentiate in PA1. However, it remains to be elucidated whether appropriate amounts of NCC migrate into PA1 in absence of Shh signal- ing, inasmuch as Smoak et al. (19) suggested that NCC were migrating along abnormal pathways in Shh mutant embryos based on the expression of NCC markers, CrabP1 and $A P 2 \alpha$.

Our analyses revealed the severe hypoplasia of the maxillary component and the proximal mandibular component, and defective midline development of PA1 in Shh mutants (Fig. 5C). The distal mandibular component where Hand2, Mhox, $D l x 3$, and $D l x 6$ are highly expressed was less affected. In the current concept of PA1 or jaw development, it has been proposed that "maxillary" and "mandibular" precursors are from distinct origins $(20,21)$, and that identities of "maxillary," "proximal mandibular," and "distal mandibular" components are controlled by specific sets of transcription factors and signaling molecules (4). Shh signaling appears to be, directly or indirectly (see discussion below), more critical for development of maxillary and proximal mandibular region, and establishment of midline structure than identities of the distal mandibular region.

Enhanced apoptosis and altered epithelial-mesenchymal interactions lead to severe hypoplasia of PA1 in Shh mutants. Our data suggest that the hypoplastic PA1 in Shh mutant embryos is mainly as a result of enhanced apoptosis in PA1 mesenchyme, indicating that Shh signaling functions in survival of PA1 mesenchymal cells. This is consistent with the observation that blocking Shh signaling in chick embryos by anti-Shh antibody results in significant increase in apoptosis in NCC-derived mesenchyme in pharyngeal arches (8), and recent experiments using lysotracker red (molecular probes) $(10,19)$. Enhanced apoptosis of mesenchymal cells was also documented in PA1 of Wnt1-Cre; Smo ${ }^{n / c}$ mouse embryos that were created by crossing mice harboring the Wntl-Cre transgene with those that contain loxP sites around the Shh receptor Smoothened (Smo) to remove Hh-signaling specifically in the cranial NCC lineage (9).

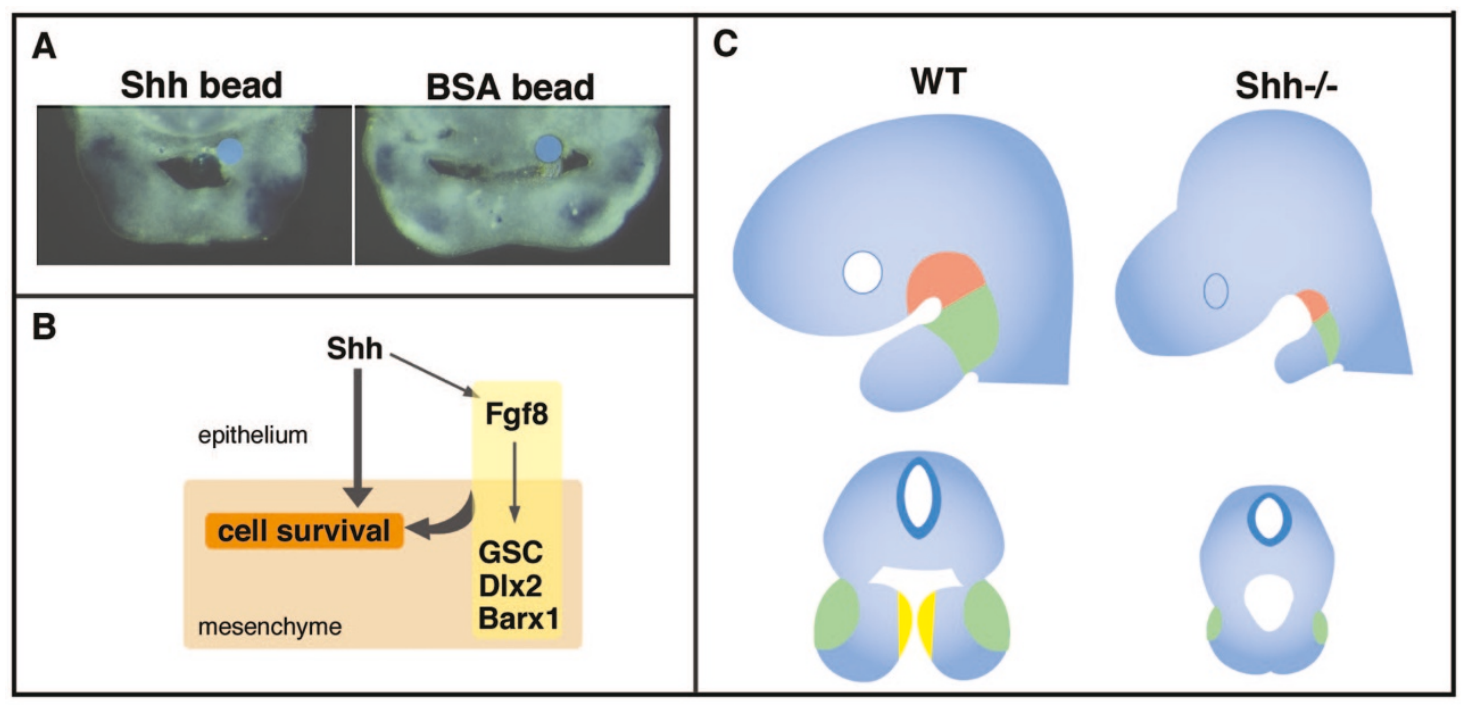

Figure 5. Shh activation of Fgf8 and proposed model for Shh signaling in PA1 development. (A) Representative Fgf8 expression in cultured PA1 explants after application of a Shh-soaked bead or control (BSA-soaked) bead. Higher level of Fgf8 expression was observed in the explants containing Shh-soaked bead. (B) Proposed model for Shh-Fgf signaling in PA1 development. Shh from epithelium may play a role in survival of mesenchymal cells in PA1 partly via Fgf8 signaling. (C) Schematic drawing of the maxillary (pink) and the proximal mandibular (green) components and the midline structure (yellow) are shown in right lateral views and transverse section of embryos at E9.5. Loss of Shh signaling results in severe hypoplasia of the maxillary and the proximal mandibular components, and defective midline development of PA1. 
Whether Shh directly acts as a survival factor for mesenchymal cells in PA1 remains to be studied. Mesenchymal cells in PA1 can directly respond to Shh signaling, as they express the Shh receptors, Smo and Patced (Ptc), and the Gli family of transcription factors that transduce Shh signaling (4,22). Jeong et al. (17) have proposed a model in which Shh signaling directly regulates growth of pharyngeal arches via combinatorial expression of several forkhead (Fox) transcription factors, and we have previously reported that Foxc2 can mediate Shh signaling in craniofacial and cardiovascular development (23). These observations suggest that Shh signaling may directly play a role in survival of mesenchymal cells and growth of PA1 through epithelial-mesenchymal interaction.

Alternatively, it is also possible that Shh signaling may promote mesenchymal cell survival in PA1 by regulating the expression of other growth factors. Our molecular analysis revealed that Fgf8 expression was down-regulated in PA1 ectoderm of Shh mutants at E9.5. Tissue-specific inactivation of $F g f 8$ by Cre-mediated recombination in the ectoderm of the PA1 leads to mesenchymal cell death around E9.5 and impairs development of PA1 (5), suggesting that Fgf8 is required, directly or indirectly, for survival of neural crest-derived mesenchyme in PA1. We also observed down-regulation of pharyngeal mesenchyme markers, Barx1, Gsc, and Dlx2, that are putative targets of Fgf8 signaling in PA1 of Shh mutant embryos. Fgf8 is normally able to induce expression of these genes, whereas Shh alone is not sufficient (3-5). In addition, other mesenchymal markers, including Hand2, Mhox, and Dlx3 were not down-regulated in Shh mutants, suggesting that there was not a generalized defect of the pharyngeal arch mesenchyme. Consistent with the idea that Shh signaling functions upstream of Fgf8 in PA1 development, Shh mutants have more severe PA1 defects than those that result from tissue-specific loss of Fgf8 function in PA1, where only the proximal region of PA1 is most severely affected (5). Our result that $F g f 8$ is activated by Shh-soaked beads in PA culture supports this idea. We therefore favor the interpretation that the PA1 phenotype of Shh mutants, at least in part, reflects a lack of Fgf8 signaling during critical epithelial-mesenchymal interactions (Fig. 5B), although we still cannot rule out the possibility that Fgf8 expression is decreased secondary to the morphologic defect.

Implication in human diseases. Mild midline defects of PA1 may lead to SMMCI. Interestingly, SMMCI is suggested as a characteristic finding in HPE patients with $S H H$ mutations (24). In addition, $S H H$ mutations have been identified not only in SMMCI with HPE, but also SMMCI without HPE $(24,25)$. These findings are consistent with a primary role of Shh in PA1 as suggested in our study.

Severe hypoplasia of PA1 in humans, on the other hand, leads to agnathia or micrognathia. Agnathia alone occurs very rarely, and is often associated with HPE and sometimes with situs inversus totalis $(25,26)$, all of which occur in the setting of $\mathrm{SHH}$ disruption in mouse and human. Moreover, Shh lies upstream of Tbxl, a major genetic determinant of DiGeorge/22q11.2 deletion syndrome that is often associated with micrognathia $(15,23)$. It is intriguing to speculate that Shh is not only a disease gene for HPE but also a genetic modifier of other human syndromes associated with agnathia/micrognathia.
Acknowledgments. The authors thank J.A. Richardson and members of the Molecular Pathology Core for assistance with histologic analysis and section in situ hybridization, S. Johnson for preparation of figures, and K. Uchida for helpful comments. We also thank C. Chiang for Shh mice; J.A. Epstein for Pax3-Cre mice; H. Yanagisawa (Dlx6, Mhox), D.E. Clouthier (Barx1), Richard Behringer (GSC), B.L.M. Hogan (Bmp4), and E.N. Meyers (Fgf8) for probes; and E.N. Meyers for mouse embryos.

\section{REFERENCES}

1. Graham A 2003Development of the pharyngeal arches. Am J Med Genet A 119:251-256

2. Richman JM, Lee SH 2003About face: signals and genes controlling jaw patterning and identity in vertebrates. Bioessays 25:554-568

3. Tucker AS, Yamada G, Grigoriou M, Pachnis V, Sharpe PT 1999Fgf-8 determines rostral-caudal polarity in the first branchial arch. Development 126:51-61

4. Tucker AS, Al Khamis A, Ferguson CA, Bach I, Rosenfeld MG, Sharpe PT 1999Conserved regulation of mesenchymal gene expression by Fgf- 8 in face and limb development. Development 126:221-228

5. Trumpp A, Depew MJ, Rubenstein JL, Bishop JM, Martin GR 1999Cre-mediated gene inactivation demonstrates that FGF8 is required for cell survival and patterning of the first branchial arch. Genes Dev 13:3136-3148

6. Massague J, Chen YG 2000Controlling TGF-beta signaling. Genes Dev 14:627-644

7. Chiang C, Litingtung Y, Lee E, Young KE, Corden JL, Westphal H, Beachy PA 1996Cyclopia and defective axial patterning in mice lacking Sonic hedgehog gene function. Nature 383:407-413

8. Ahlgren SC, Bronner-Fraser M 1999Inhibition of sonic hedgehog signaling in vivo results in craniofacial neural crest cell death. Curr Biol 9:1304-1314

9. Jeong J, Mao J, Tenzen T, Kottmann AH, McMahon AP 2004Hedgehog signaling in the neural crest cells regulates the patterning and growth of facial primordia. Genes Dev 18:937-51

10. Moore-Scott BA, Manley NR 2005Differential expression of Sonic hedgehog along the anterior-posterior axis regulates patterning of pharyngeal pouch endoderm and pharyngeal endoderm-derived organs. Dev Biol 278:323-335

11. Epstein JA, Li J, Lang D, Chen F, Brown CB, Jin F, Lu, Thomas M, Liu E, Wessels A, Lo CW 2000Migration of cardiac neural crest cells in Splotch embryos. Development 127:1869-1878

12. Soriano P 1999 Generalized lacZ expression with the ROSA26 Cre reporter strain Nat Genet 21:70-71

13. Yamagishi H, Olson EN, Srivastava D 2000The basic helix-loop-helix transcription factor, dHAND, is required for vascular development. J Clin Invest 105:261-270

14. Yamagishi H, Yamagishi C, Nakagawa O, Harvey RP, Olson EN, Srivastava D 2001The combinatorial activities of $\mathrm{Nkx} 2.5$ and dHAND are essential for cardiac ventricle formation. Dev Biol 239:190-203

15. Garg V, Yamagishi C, Hu T, Kathiriya IS, Yamagishi H, Srivastava D 2001Tbx1, a DiGeorge syndrome candidate gene, is regulated by sonic hedgehog during pharyngeal arch development. Dev Biol 235:62-73

16. Zakin L, DeRobertis EM 2004Inactivation of mouse Twisted gastrulation reveals its role in promoting Bmp4 activity during forebrain development. Development 131:413-424

17. Liu W, Selever J, Wang D, Lu MF, Moses KA, Schwartz RJ, Martin JF 2004Bmp4 signaling is required for outflow-tract septation and branchial-arch artery remodeling. Proc Natl Acad Sci U S A 101:4489-4494

18. ten Berge D, Brouwer A, Korving J, Reijnen MJ, van Raaij EJ, Verbeek F, Gaffield W, Meijlink F 2001Prx1 and Prx2 are upstream regulators of sonic hedgehog and control cell proliferation during mandibular arch morphogenesis. Development 128:2929-2938

19. Washington Smoak I, Byrd NA, Abu-Issa R, Goddeeris MM, Anderson R, Morris J, Yamamura K, Klingensmith J, Meyers EN 2005Sonic hedgehog is required for cardiac outflow tract and neural crest cell development. Dev Biol 283:357-372

20. Lee SH, Bedard O, Buchtova M, Fu K, Richman JM 2004A new origin for the maxillary jaw. Dev Biol 276:207-224

21. Cerny R, Lwigale P, Ericsson R, Meulemans D, Epperlein HH, Bronner-Fraser M 2004Developmental origins and evolution of jaws: new interpretation of "maxillary" and "mandibular". Dev Biol 276:225-236

22. McMahon AP, Ingham PW, Tabin CJ 2003Developmental roles and clinical significance of hedgehog signaling. Curr Top Dev Biol 53:1-114

23. Yamagishi H, Maeda J, Hu T, McAnally J, Conway SJ, Kume T, Meyers EN, Yamagishi C, Srivastava D 2003Tbx1 is regulated by tissue-specific forkhead proteins through a common Sonic hedgehog-responsive enhancer. Genes Dev 17:269-281

24. Nanni L, Ming JE, Du Y, Hall RK, Aldred M, Bankier A, Muenke M 2001SHH mutation is associated with solitary median maxillary central incisor: a study of 13 patients and review of the literature. Am J Med Genet 102:1-10

25. Garavelli L, Zanacca C, Caselli G, Banchini G, Dubourg C, David V, Odent S, Gurrieri F, Neri G 2004Solitary median maxillary central incisor syndrome: clinical case with a novel mutation of sonic hedgehog. Am J Med Genet A 127:93-95

26. Leech RW, Bowlby LS, Brumback RA, Schaefer Jr GB 1988Agnathia, holoprosencephaly, and situs inversus: report of a case. Am J Med Genet 29:483-490 